\title{
Experimenting with computing and in computing: Stretching the traditional notion of experimentation
}

\author{
Viola Schiaffonati ${ }^{1}$ \\ ${ }^{1}$ Artificial Intelligence and Robotics Laboratory \\ Dipartimento di Elettronica, Informazione e Bioingegneria \\ Politecnico di Milano \\ viola.schiaffonati@polimi.it
}

\begin{abstract}
Experimentation represents today a 'hot' topic in computing. If experiments made with the support of computers, such as computer simulations, have received increasing attention from philosophers of science and technology, questions such as "what does it mean to do experiments in computer science and engineering and what are their benefits?" emerged only recently as central in the debate over the disciplinary status of computing. In this work I argue that an extension of the traditional notion of controlled experiment is necessary to give reasons for the different experimental practices encountered in computing and using computing tools, such as computer simulations. Taking inspiration from the discussion on exploratory experimentation in the philosophy of science experimentation that is not theory-driven - I introduce the idea of explorative experiments that can contribute to enlarge the debate about the nature and role of experimental methods in computing. Moreover, I stress that, when experiments are explorative, control should be intended in a posteriori form, in opposition to the a priori form that usually takes place in traditional experimental contexts.
\end{abstract}

\section{Experiments and computing: Not only simulations}

Experimentation represents today a 'hot' topic in computing. Not only experiments made with the support of computers, such as computer simulations, play an essential role in every domain of science and technology, but also questions such as "what does it mean to make experiments in computer science and engineering and what are their benefits?" have recently taken a center stage in the debate over the disciplinary status of computing. However, if the experimental capabilities of computer simulations have received increasing attention from philosophers of science, the same attention has not been devoted to analyze the notion of experiment in the realm of computing. In this work I argue that an extension of the traditional notion of controlled experiment is necessary to give reasons for the different experimental practices encountered in computing and using computing tools, such as computer simulations. I rely in particular on examples from autonomous robotics, an area of computer engineering where the interplay between the scientific and engineering aspects of computing is strong.
It is undoubtedly evident that science has entered what has been called the 'age of computer simulation' (Winsberg, 2010); the massive use of computer simulations in virtually every domain of science has drawn attention to their epistemological justification: if computer simulations started as tools to build tractable models for solving the equations provided by theories, nowadays their role expanded and, besides dealing with the construction of models of greater and greater complexity, computer simulations can be used to increase the exploration opportunities. This is in accordance with the idea of 'modeling from above' (Fox Keller, 2003) and 'modeling from the ground up' (MacLeod and Nersessian, 2013), where the theoretical model supporting the simulation is under construction and shaped by the simulation results themselves. Accordingly, a different relation between theory and experiment emerges, where the latter actively participates in the settling of the former, instead of aiming only at testing or rejecting the theory itself.

Recently the experimental properties of computer simulations have been examined, and philosophers have begun to consider in what sense, if any, computer simulations are experiments (see (Winsberg, 2013) for a detailed analysis of this debate). Positions range from a full acceptance of the identity thesis ("Computer simulation studies are literally instances of experiments") to its rejection in different degrees. Computer simulations can be used as experiments in the case in which the purposes of simulation and those of experiment coincide, but they are not necessarily experiments: it is perfectly plausible to have computer simulations at work that do not have any experimental purpose (think for example of simulations adopted for didactical purposes).

The possibility of using simulations as experiments resides in the ability (and in the necessity) they both possess of controlling the features under investigation, thus implementing the original idea of experiment as a controlled experience. The source of credibility for the models used in the different cases of simulations as experiments greatly varies: if the case in which the credentials are provided by the theoretical ancestors on which simulations are based is not problematic, the 'modeling from above' or 'from the ground up' practices require an epistemological justification for their use. Indeed, the use of computer simulations also in those fields that do not have secure theoretical foundations and/or in which data are sparse has reshaped the way experimental 
results are considered reliable. In these contexts experiments (in the form of computer simulations) cannot be considered as pure controlled experiences anymore, but are better conceived as explorations, where the theoretical background is progressively shaped by simulation results.

When moving from the analysis of experiments made with computing (simulations) to experiments made in computing, a closer look on whether and to what extent the traditional experimental protocol can be applied to computing provides evidence that the same explorative approach used in computer simulations is already at work in this context, even if it is not yet properly conceptualized. Taking inspiration from the discussion on exploratory experimentation in the philosophy of science - experimentation that is not theory-driven - and from the broad notion of directly action-guiding experiment (Hansson, 2016), I advance the idea of explorative experiments that could contribute to enlarge the debate about the nature and role of experimental methods in computing. By investigating autonomous robotics in particular, it is evident that the traditional notion of experimentation cannot be always applied as such to computer engineering experimental practices and that the notion of explorative experiment is a good candidate to be considered. I suggest, moreover, that, when experiments are explorative, control should be intended in a posteriori form, in opposition to the a priori form that usually takes place in traditional experimental contexts.

In general an experiment is a set of observations and actions, performed in a controlled context, to test theories and to provide a basis for scientific knowledge (Hacking, 1983; Franklin, 1986; Radder, 2003). Control, intended as an active manipulation of the phenomena under investigation where the choice of the factors to be controlled is critical, is usually considered a central feature of experimentation so that experiments are also labelled 'controlled experiments'. By explorative experiments I mean a form of investigation of novel and interesting ideas or techniques without the typical constraints of rigorous experimental methodologies. These are experiments that are driven by the desire of investigating the realm of possibilities pertaining to the functioning of an artefact and its interaction with the environment in the absence of a proper theory or theoretical background. So hypotheses cannot be clearly stated and, even if the ultimate goal is to acquire knowledge about the performance of the artefacts under investigation and to find out proper concepts to formulate possible regularities, the experimenter is not in full control of the experimental setting due to the impossibility of anticipating all the plausible outcomes. Therefore, when experiments are explorative, control should be intended in a posteriori form, in opposition to the a priori form of the traditional experimental contexts. If in the latter experimental factors are in control of the experimenter in a sort of anticipation of the scenario to be tested, in the former the possibility of full anticipation disappears and control is in part carried out after the artefact has been inserted into society. This is in line with the crisis of the traditional notion of experimental control that has been recently evidenced by Peter Kroes (2016) in his analysis of experiments with new technologies in socio-technical systems. The traditional control paradigm is based on two assumptions: the experimenter is not part of the system on which the experiment is performed and (s)he is in control of the independent variables and of the experimental set-up. Accordingly, the experimenter is able to intervene both by changing these variables to evaluate their influence on the dependent ones and by varying the experimental set-up. This traditional control paradigm becomes problematic, and a consequent shift in the notions of intervention and control is observed, when considering new technologies as sociotechnical systems, namely as hybrid systems composed of natural objects, technical artefacts, human actors, and social entities. The idea of controlling the experimental system from a center of command and control that is outside the system becomes highly problematic because the distinction between the experimental system and its environment is critical and the environment is complex due to the co-presence of technical artefacts and natural and social elements. Hence controlling these systems not only involves technical artefacts and social elements, but also problematizes the drawing of the line between the experimental system and its environment.

\section{References}

Franklin, A. (1986). The Neglect of Experiment. Cambridge University Press, Cambridge.

Hacking, I. (1983). Representing and Intervening. Cambridge University Press, New York.

Hansson, S.O. (2016). Experiments: Why and How? Science and Engineering Ethics, 22(3), 613-632.

Keller, E.F. (2002). Making Sense of Life: Explaining Biological Development with Models, Metaphors, and Machines. Harvard University Press, Cambridge, MA.

Kroes, P. (2016). Experiments on Socio-Technical Systems: The Problem of Control. Science and Engineering Ethics, 22(3), 633-645.

McLeod, M. and Nersessian, N. J. (2013). Building Simulations from the Ground Up: Modeling and Theory in Systems Biology. Philosophy of Science, 80(4): 533-556.

Radder, H. editor (2003). The Philosophy of Scientific Experimentation. The University of Pittsburgh Press, Pittsburgh.

Winsberg, E. (2010). Science in the Age of Computer Simulations. The University of Chicago Press, Chicago and London.

Winsberg, E. (2013). Computer Simulations in Science. The Stanford Encyclopedia of Philosophy, E. N. Zalta (ed.), URL = http://plato.stanford.edu/archives/sum2013/entries/simulationsscience/. 